PROCEEDINGS OF THE

AMERICAN MATHEMATICAL SOCIETY

Volume 133, Number 3, Pages 787-794

S 0002-9939(04)07635-X

Article electronically published on September 8, 2004

\title{
MULTIPLICITY RESULTS FOR A CLASS OF SUPERLINEAR ELLIPTIC PROBLEMS
}

\author{
DAVID G. COSTA AND ZHI-QIANG WANG
}

(Communicated by David S. Tartakoff)

\begin{abstract}
We study a class of superlinear elliptic problems $-\Delta u=\lambda f(u)$ under the Dirichlet boundary condition on a bounded smooth domain in $\mathbb{R}^{N}$. Assuming that the nonlinearity $f(u)$ is superlinear in a neighborhood of $u=0$, we study the dependence of the number of signed and sign-changing solutions on the parameter $\lambda$.
\end{abstract}

\section{INTRODUCTION}

In this paper we consider the question of multiplicity of both signed and signchanging solutions for the one-parameter family of elliptic problems $\left(P_{\lambda}\right)$

$$
\left\{\begin{aligned}
-\Delta u & =\lambda f(u) \text { in } \Omega \\
u & =0 \text { on } \partial \Omega
\end{aligned}\right.
$$

where $\lambda>0$ is a parameter, $\Omega$ is a bounded smooth domain in $\mathbb{R}^{N}(N \geq 3)$, and the nonlinearity $f: \mathbb{R} \longrightarrow \mathbb{R}$ is a function of class $C^{1}$ satisfying the following conditions:

$\left(f_{1}\right)$ there exists $\gamma \in\left(2,2^{*}\right)$ such that $\lim \sup _{|u| \rightarrow 0} \frac{f(u) u}{|u|^{\gamma}}<+\infty$,

$\left(f_{2}\right)$ there exists $\beta \in\left(2,2^{*}\right)$ such that $\liminf _{|u| \rightarrow 0} \frac{F(u)}{|u|^{\beta}}>0$,

$\left(f_{3}\right)$ there exists $\mu \in\left(2,2^{*}\right)$ such that $u f(u) \geq \mu F(u)>0$ for $0 \neq|u|$ small,

$\left(f_{4}\right) f(-u)=-f(u) \forall|u| \leq \delta$ (for some $\delta>0$ ).

Here $2^{*}=\frac{2 N}{N-2}$ is the critical Sobolev exponent and $F(u)=\int_{0}^{u} f(t) d t$. As is well known, if $f$ were assumed to be superlinear at infinity (in the sense that $\left(f_{1}\right)$ and $\left(f_{2}\right)$ hold as $|u| \rightarrow \infty$ and $\left(f_{3}\right)$ holds for $|u|$ large), then the associated energy functional

$$
I(u)=\frac{1}{2} \int_{\Omega}|\nabla u|^{2} d x-\lambda \int_{\Omega} F(u) d x
$$

would be of class $C^{1}$ on $H_{0}^{1}(\Omega)$ and satisfy the Palais-Smale condition, with its critical points being precisely the solutions of problem $\left(P_{\lambda}\right)$. Here, the assumptions $\left(f_{1}\right)-\left(f_{3}\right)$ we make on the nonlinearity $f(u)$ refer solely to its behavior in a neighborhood of $u=0$, and we will show that they suffice for the existence of three solutions of problem $\left(P_{\lambda}\right)$ when $\lambda$ is large. In addition, if $f(u)$ is odd near $u=0$, the number of sign-changing solutions of $\left(P_{\lambda}\right)$ gets arbitrarily large together with

Received by the editors October 24, 2003.

2000 Mathematics Subject Classification. Primary 35J20.

(C)2004 American Mathematical Society Reverts to public domain 28 years from publication 
$\lambda$. With global superlinear conditions these results were known in [1, 3, 4, 5]. More precisely, we will prove the following results.

Theorem 1. Assume conditions $\left(f_{1}\right)-\left(f_{3}\right)$ are satisfied. Then there exists $\Gamma>0$ depending only on $\gamma$ such that, if $(\beta-\gamma) \Gamma<1$ is satisfied, problem $\left(P_{\lambda}\right)$ has at least one positive solution, one negative solution, and a sign-changing solution for all $\lambda$ sufficiently large.

Theorem 2. Assume conditions $\left(f_{1}\right)-\left(f_{4}\right)$ are satisfied. Then there exists $\Gamma>0$ depending only on $\gamma$ such that, if $(\beta-\gamma) \Gamma<1$ is satisfied then, for any given $k \geq 1$, problem $\left(P_{\lambda}\right)$ has $k$ pairs of solutions $\pm v_{i}, i=1, \ldots, k$, with $\left|v_{i}\right|_{\infty} \leq \delta$, provided $\lambda$ is sufficiently large. Moreover, $\pm v_{i}$ for $i=2, \ldots, k$, are sign-changing solutions.

Our approach is inspired by the results of Costa-Tehrani 2] and is based on the fact that we can show an a priori bound of the form

$$
|u|_{\infty} \leq C \lambda^{-\epsilon}, \epsilon>0
$$

for a class of solutions of $\left(P_{\lambda}\right)$ with energy estimates given by minimax methods. The energy estimates for sign-changing solutions rely upon the minimax procedure of Li-Wang [4] for constructing nodal critical points.

The organization of this paper is as follows. Section 1 is reserved for setting the framework and establishing some preliminary results. Theorems 1 and 2 are proved in Section 2.

\section{Preliminary Results}

Throughout the paper we denote the $H_{0}^{1}$-norm by $\|\cdot\|$ and the $L^{r}$-norm by $|\cdot|_{r}$, $1 \leq r \leq \infty$. Also, sometimes we denote various positive constants by the same letter $C_{*}$.

We start by observing that $\left(f_{2}\right)$ and $\left(f_{1}\right)$ imply the existence of constants $C_{0}, C_{1}$ $>0$ such that

$$
\begin{aligned}
& F(u) \geq C_{0}|u|^{\beta}, \\
& F(u) \leq C_{1}|u|^{\gamma},
\end{aligned}
$$

for $|u|$ small. Now, let $\rho(t)$ be an even cut-off function satisfying $\rho(t) \equiv 1$ if $|t| \leq \delta$, $\rho(t) \equiv 0$ if $|t| \geq 2 \delta, t \rho^{\prime}(t) \leq 0$, and $\left|t \rho^{\prime}(t)\right| \leq \frac{2}{\delta}$, where $0<\delta<\frac{1}{2}$ is chosen such that (1.1), (1.2) and $\left(f_{3}\right)$ hold for $|u| \leq 2 \delta$.

Lemma 1.1. Define $\widetilde{F}(u)=\rho(u) F(u)+(1-\rho(u)) F_{\infty}(u)$, where $F_{\infty}(u):=C_{1}|u|^{\gamma}$. Then

$$
u \widetilde{F}^{\prime}(u) \geq \theta \widetilde{F}(u)>0
$$

for all $u \neq 0$, where $\theta=\min \{\mu, \gamma\}$.

Proof. We have

$$
\widetilde{F}^{\prime}(u)=\rho(u) f(u)+(1-\rho(u)) F_{\infty}^{\prime}(u)+\rho^{\prime}(u) F(u)-\rho^{\prime}(u) F_{\infty}(u)
$$


so that

$$
\begin{gathered}
\theta \widetilde{F}(u)=\theta \rho(u) F(u)+\theta(1-\rho(u)) F_{\infty}(u) \\
\leq \rho(u) \frac{\theta}{\mu} f(u) u+(1-\rho(u)) \frac{\theta}{\gamma} F_{\infty}^{\prime}(u) u \\
+\rho^{\prime}(u) u F(u)-\rho^{\prime}(u) u F_{\infty}(u)-\rho^{\prime}(u) u F(u)+\rho^{\prime}(u) u F_{\infty}(u) \\
\leq u \widetilde{F}^{\prime}(u)+\rho^{\prime}(u) u\left(F_{\infty}(u)-F(u)\right) \leq u \widetilde{F}^{\prime}(u) .
\end{gathered}
$$

Now let us consider the modified equation of $\left(P_{\lambda}\right)$ given by $\left(\widetilde{P}_{\lambda}\right)$

$$
\left\{\begin{aligned}
-\Delta u & =\lambda \widetilde{F}^{\prime}(u) \text { in } \Omega \\
u & =0 \text { on } \partial \Omega
\end{aligned}\right.
$$

The corresponding functional

$$
\widetilde{I}_{\lambda}(u)=\frac{1}{2} \int_{\Omega}|\nabla u|^{2} d x-\lambda \int_{\Omega} \widetilde{F}(u) d x, \quad u \in E:=H_{0}^{1}(\Omega),
$$

is of class $C^{2}$, and its critical points are the solutions of $\left(\widetilde{P}_{\lambda}\right)$. We note that critical points of $\left(\widetilde{I}_{\lambda}\right)$ with $L^{\infty}$-norm less than or equal to $\delta$ are also solutions of the original problem $\left(P_{\lambda}\right)$.

Lemma 1.2. The functional $\widetilde{I}_{\lambda}$ satisfies $(P S)$ on $E$.

Proof. This is standard in view of Lemma 1.1 and the fact that $\gamma<2^{*}$.

Lemma 1.3. Let $u \in E$ be a critical point of $\widetilde{I}_{\lambda}$. Then

$$
\|u\|^{2} \leq \frac{2 \theta}{\theta-2} \widetilde{I}_{\lambda}(u) .
$$

Proof. This estimate readily follows from Lemma 1.1 and

$$
\frac{1}{2}\|u\|^{2}-\lambda \int_{\Omega} \widetilde{F}(u) d x=\widetilde{I}_{\lambda}(u),\|u\|^{2}-\lambda \int_{\Omega} \widetilde{F}^{\prime}(u) u d x=0 .
$$

Now, let $0<\lambda_{1}<\lambda_{2} \leq \lambda_{3} \leq \ldots$ and $\phi_{1}, \phi_{2}, \phi_{3}, \ldots$ denote the eigenvalues and corresponding eigenfunctions of $-\Delta$ on $H_{0}^{1}(\Omega)$. Also, for $k=1,2, \ldots$, let

$$
Y_{k}:=\operatorname{span}\left\{\phi_{1}, \ldots, \phi_{k}\right\}, \quad Z_{k}:=\overline{\operatorname{span}}\left\{\phi_{k}, \phi_{k+1}, \ldots\right\} .
$$

Lemma 1.4. Let $b_{k, \lambda}=\sup _{u \in Y_{k}} \widetilde{I}_{\lambda}(u)$. Then

$$
b_{k, \lambda} \leq C_{*} C_{k} \lambda^{-\frac{2}{\beta-2}}
$$

where $C_{*}>0$ depends only on $\gamma, \beta$ and $\Omega$ and $C_{k}:=\lambda_{k}^{\frac{\beta}{\beta-2}}+\lambda_{k}^{\frac{\gamma}{\gamma-2}}$.

Proof. We recall that $\delta>0$ was chosen so that

$$
\begin{gathered}
\widetilde{F}(u) \geq F(u) \geq C_{0}|u|^{\beta} \quad \text { for } \quad|u| \leq 2 \delta, \\
\widetilde{F}(u)=F_{\infty}(u)=C_{1}|u|^{\gamma} \quad \text { for } \quad|u| \geq 2 \delta .
\end{gathered}
$$


For $u \in E$, denote $\Omega_{1}=\{x|| u \mid \geq 2 \delta\}, \Omega_{2}=\{x|| u \mid<2 \delta\}$, and let $u_{1}=u \mid \Omega_{1}$, $u_{2}=u \mid \Omega_{2}$. In view of Hölder's inequality we obtain

$$
\begin{gathered}
\int_{\Omega} \widetilde{F}\left(u_{2}\right) d x \geq C_{*}\left|u_{2}\right|_{2}^{\beta}, \\
\int_{\Omega} \widetilde{F}\left(u_{1}\right) d x \geq C_{*}\left|u_{1}\right|_{2}^{\gamma},
\end{gathered}
$$

so that, for $u \in Y_{k}$, it follows that

$$
\begin{gathered}
\widetilde{I}_{\lambda}(u) \leq \frac{\lambda_{k}}{2}|u|_{2}^{2}-\lambda C_{*}\left|u_{1}\right|_{2}^{\gamma}-\lambda C_{*}\left|u_{2}\right|_{2}^{\beta} \\
=\frac{\lambda_{k}}{2}\left|u_{1}\right|_{2}^{2}-\lambda C_{*}\left|u_{1}\right|_{2}^{\gamma}+\frac{\lambda_{k}}{2}\left|u_{2}\right|_{2}^{2}-\lambda C_{*}\left|u_{2}\right|_{2}^{\beta} \\
\leq C_{*} \lambda_{k}^{\frac{\gamma}{\gamma-2}} \lambda^{-\frac{2}{\gamma-2}}+C_{*} \lambda_{k}^{\frac{\beta}{\beta-2}} \lambda^{-\frac{2}{\beta-2}} .
\end{gathered}
$$

Since $2<\gamma \leq \beta<2^{*}$, we obtain the following estimate:

$$
b_{k, \lambda} \leq C_{*} C_{k} \lambda^{-\frac{2}{\beta-2}},
$$

where $C_{k}:=\lambda_{k}^{\frac{\beta}{\beta-2}}+\lambda_{k}^{\frac{\gamma}{\gamma-2}}$.

\section{Proofs of the theorems}

As is well known, $\left(\widetilde{P}_{\lambda}\right)$ has a positive solution $u_{1, \lambda}$ and a negative solution $u_{2, \lambda}$ obtained through an application of the Mountain-Pass Theorem [1] to the functionals

and

$$
\widetilde{I}_{1, \lambda}(u)=\frac{1}{2} \int_{\Omega}|\nabla u|^{2} d x-\lambda \int_{\Omega} \widetilde{F}_{1}(u) d x, \quad u \in E:=H_{0}^{1}(\Omega)
$$

$$
\widetilde{I}_{2, \lambda}(u)=\frac{1}{2} \int_{\Omega}|\nabla u|^{2} d x-\lambda \int_{\Omega} \widetilde{F}_{2}(u) d x, \quad u \in E:=H_{0}^{1}(\Omega),
$$

respectively, where $\widetilde{F}_{1}(u)=\widetilde{F}(u)$ if $u>0, \widetilde{F}_{1}(u) \equiv 0$ if $u \leq 0$, and $\widetilde{F}_{2}(u)=\widetilde{F}(u)$ if $u<0, \widetilde{F}_{2}(u) \equiv 0$ if $u \geq 0$. The corresponding critical values are given by

$$
\widetilde{d}_{j, \lambda}=\inf _{h \in \Gamma_{j}} \sup _{0 \leq t \leq 1} \widetilde{I}_{j, \lambda}(h(t)), \quad j=1,2,
$$

where $\Gamma_{j}=\left\{h \in C([0,1], E) \mid h \neq 0, \widetilde{I}_{j, \lambda}(h(1)) \leq 0\right\}$. It is clear that

$$
\tilde{d}_{j, \lambda} \leq d_{j, \lambda}
$$

where

$$
d_{1, \lambda}:=\inf _{u>0} \sup _{0 \leq t<\infty} \widetilde{I}_{\lambda}(t u), d_{2, \lambda}:=\inf _{u<0} \sup _{0 \leq t<\infty} \widetilde{I}_{\lambda}(t u) .
$$

Lemma 2.1. $\widetilde{d}_{j, \lambda} \leq C_{*} \lambda^{-\frac{2}{\beta-2}}$ for $j=1,2$ and $\lambda>0$ large.

Proof. From Lemma 1.3 and (2.1), we have the estimates

$$
\left\|u_{j, \lambda}\right\| \leq C_{*} \sqrt{d_{j, \lambda}}, \quad j=1,2 .
$$

Let us consider $u_{1, \lambda}$ since the same argument applies to $u_{2, \lambda}$. Recalling from (1.6) that $\widetilde{F}(u) \geq C_{0}|u|^{\beta}$ for $|u| \leq 2 \delta$ and defining

$$
J_{\lambda}(u)=\frac{1}{2} \int_{\Omega}|\nabla u|^{2} d x-\lambda \int_{\Omega} C_{0}|u|^{\beta} d x
$$


we have

$$
\begin{aligned}
& d_{1, \lambda}=\inf _{u>0} \sup _{0 \leq t<\infty} \widetilde{I}_{\lambda}(t u) \leq \inf _{0<u \leq 2 \delta} \sup _{0 \leq t<\infty} \widetilde{I}_{\lambda}(t u) \\
& \leq \inf _{0<u \leq 2 \delta} \sup _{0 \leq t<\infty} J_{\lambda}(t u)=\inf _{u \neq 0} \sup _{0 \leq t<\infty} J_{\lambda}(t u)
\end{aligned}
$$

where the last equality holds provided $\lambda>0$ is sufficiently large so that the ground states of $J_{\lambda}(u)$ have $L^{\infty}$-norm less than $2 \delta$. In fact, we obtain through straightforward calculations as in [2, Thm. 1.1] that

$$
\inf _{u \neq 0} \sup _{0 \leq t<\infty} J_{\lambda}(t u) \leq C_{*} \lambda^{-\frac{2}{\beta-2}} .
$$

Therefore, keeping in mind $(\underline{2.3})$ and $(2.2)$ above, we conclude that

$$
d_{1, \lambda} \leq C_{*} \lambda^{-\frac{2}{\beta-2}}
$$

and

$$
\left\|u_{1, \lambda}\right\| \leq C_{*} \lambda^{-\frac{1}{\beta-2}}
$$

for $\lambda>0$ large. It follows by Sobolev's inequality that

$$
\left|u_{1, \lambda}\right|_{2^{*}} \leq C_{*} \lambda^{-\frac{1}{\beta-2}}
$$

for $\lambda>0$ large. The proof is complete in view of (2.4) and (2.1).

Lemma 2.2. Let $u_{\lambda}$ be solutions of $\left(P_{\lambda}\right)$ satisfying $\widetilde{I}_{\lambda}\left(u_{\lambda}\right) \leq C_{*} \lambda^{-\frac{2}{\beta-2}}$. Then there is an integer $m$ depending only on $\gamma$ such that for all $\lambda \geq 1$,

$$
\left|u_{\lambda}\right|_{\infty} \leq C_{*} \lambda^{-\frac{1}{\beta-2}} \lambda^{\frac{\beta-\gamma}{\beta-2} \sum_{i=0}^{m-1}(\gamma-1)^{i}} .
$$

Proof. Since the cut-off function $\rho(t)$ satisfies $\left|\rho^{\prime}(t) t\right| \leq 2 / \delta$, we obtain from (1.3) that

$$
\left|\widetilde{F}^{\prime}\left(u_{\lambda}\right)\right| \leq C_{*}\left|u_{\lambda}\right|^{\gamma-1}
$$

hence

$$
\widetilde{F}^{\prime}\left(u_{\lambda}\right) \in L^{\frac{2^{*}}{\gamma-1}}(\Omega) .
$$

Now, if $r_{1}=\frac{2^{*}}{\gamma-1}$, then $L^{r_{1}}$-estimates give

$$
\begin{aligned}
\left\|u_{\lambda}\right\|_{W^{2, r_{1}}} & \leq C_{*}\left(\left|u_{\lambda}\right|_{r_{1}}+\lambda\left|\widetilde{F}^{\prime}\left(u_{\lambda}\right)\right|_{r_{1}}\right) \\
& \leq C_{*}\left(C_{*}\left|u_{\lambda}\right|_{2^{*}}+\lambda C_{*}\left|u_{\lambda}\right|_{2^{*}}^{\gamma-1}\right) \\
& \leq C_{*}\left(\lambda^{-\frac{1}{\beta-2}}+\lambda \lambda^{-\frac{\gamma-1}{\beta-2}}\right) \\
& \leq C_{*}\left(\lambda^{-\frac{1}{\beta-2}}+\lambda^{-\frac{1}{\beta-2}} \lambda^{\frac{\beta-\gamma}{\beta-2}}\right)
\end{aligned}
$$

hence

$$
\left|u_{\lambda}\right|_{s_{1}} \leq C_{*}\left(\lambda^{-\frac{1}{\beta-2}} \lambda^{\frac{\beta-\gamma}{\beta-2}}\right)
$$


where $\frac{1}{s_{1}}=\frac{1}{r_{1}}-\frac{2}{N}$. Note that $s_{1}>2^{*}$ since $\gamma<2^{*}$. Next, letting $r_{2}=\frac{s_{1}}{\gamma-1}$ and using $L^{r_{2}}$-estimates, we obtain

$$
\begin{aligned}
\left\|u_{\lambda}\right\|_{W^{2, r_{2}}} & \leq C_{*}\left(\left|u_{\lambda}\right|_{r_{2}}+\lambda\left|\widetilde{F}^{\prime}\left(u_{\lambda}\right)\right|_{r_{2}}\right) \\
& \leq C_{*}\left(C_{*}\left|u_{\lambda}\right|_{s_{1}}+\lambda C_{*}\left|u_{\lambda}\right|_{s_{1}}^{\gamma-1}\right) \\
& \leq C_{*}\left(\lambda^{-\frac{1}{\beta-2}} \lambda^{\frac{\beta-\gamma}{\beta-2}}+\lambda \lambda^{-\frac{\gamma-1}{\beta-2}} \lambda^{\frac{(\gamma-1)(\beta-\gamma)}{\beta-2}}\right) \\
& \leq C_{*}\left(\lambda^{-\frac{1}{\beta-2}} \lambda^{\frac{\beta-\gamma}{\beta-2}}+\lambda^{-\frac{1}{\beta-2}} \lambda^{\frac{\gamma(\beta-\gamma)}{\beta-2}}\right) .
\end{aligned}
$$

Iterating $m \geq 1$ times yields

$$
\left\|u_{\lambda}\right\|_{W^{2, r_{m}}} \leq C_{*} \lambda^{-\frac{1}{\beta-2}} \lambda^{\frac{\beta-\gamma}{\beta-2} \sum_{i=0}^{m-1}(\gamma-1)^{i}},
$$

and the result follows by taking $m$ so that $r_{m}>\frac{N}{2}$.

Remark 2.3. The number $m$ of iterations needed to have $W^{2, r_{m}}(\Omega) \subset L^{\infty}(\Omega)$ in Lemma 2.2 depends only on $N$ and $\gamma$. In other words, since the space dimension $N$ is given, the positive number

$$
\Gamma:=\sum_{i=0}^{m-1}(\gamma-1)^{i}
$$

above depends solely on $\gamma$. Therefore, if $\beta \geq \gamma$ is such that

$$
(\beta-\gamma) \Gamma<1 \text {, }
$$

we get a negative exponent for $\lambda$ in (2.7).

Proof of Theorem 1. Let $2<\gamma \leq \beta<2^{*}$ be such that $(\beta-\gamma) \Gamma<1$, where $\Gamma>0$ was defined in Remark 2.3 . Then

$$
\left|u_{j, \lambda}\right|_{\infty} \leq C_{*} \lambda^{-\frac{1-(\beta-\gamma) \Gamma}{\beta-2}}
$$

where the exponent of $\lambda$ is negative, so that there exists $\Lambda_{0}>0$ such that

$$
C_{*} \lambda^{-\frac{1-(\beta-\gamma) \Lambda}{\beta-2}} \leq \delta
$$

for all $\lambda \geq \Lambda_{0}$. It follows that $u_{1, \lambda}>0$ and $u_{2, \lambda}<0$ are solutions of our original problem $\left(P_{\lambda}\right)$ for all $\lambda \geq \Lambda_{0}$.

Since $f$ is assumed to be of class $C^{1}$ and $\inf _{s \in \mathbb{R}} \widetilde{F}^{\prime}(s)>-\infty$ by construction, we also prove that there is a sign-changing solution for all $\lambda$ large. We employ the method in [4]. On $E$, let us define

$$
P_{E}=\{u \in E \mid u(x) \geq 0, \text { a.e. in } \Omega\},
$$

which is a closed convex cone. Then, the Banach space $X=C_{0}^{1}(\Omega)$ is densely embedded in $E$, and

$$
P=P_{E} \cap X
$$

is a closed convex cone in $X$. Furthermore, $P=\stackrel{\circ}{P} \cup \partial P$ under the topology of $X$, i.e., there exist interior points in $P$. So, as in [4, Section 3], we may define a partial order relation in $X: u, v \in X, u>v \Longleftrightarrow u-v \in P \backslash\{0\} ; u \gg v \Longleftrightarrow u-v \in \stackrel{\circ}{P}$. We also define $W=P \cup(-P)$.

We follow the arguments of Example 3.2 and Corollary 3.2 in [4]. On $Y_{2}$ consider $Q=\left\{u=s \phi_{1}+t \phi_{2}|| s \mid \leq R, 0 \leq t \leq R,\|u\| \leq R\right\}$ and for $0<r<R$, $T=\left\{u \in Z_{2} \mid\|u\|=r\right\}$. Note that the boundary of $Q$ contains two parts: $Q_{1}$ is 
the part contained in $Y_{1}$ and $Q_{2}$ is the part such that $\|u\|=R$. From the conditions on $\widetilde{F}$ we may assume $R>0$ is large enough so that $\widetilde{I}_{\lambda}(u) \leq 0$ for all $u \in Y_{2}$ with $\|u\|=R$ and for all $\lambda \geq 1$. Also we may assume $r>0$ is small enough so that $\widetilde{I}_{\lambda}(u)>0$ for all $u \in T$ and for all $\lambda \geq 1$. Define

$$
\begin{gathered}
\Gamma=\left\{h \in C(Q, X) \mid h\left(Q_{1}\right) \in W, h(u)=u, \text { for } u \in Q_{2}\right\}, \\
c_{\lambda}=\inf _{h \in \Gamma} \sup _{h(Q) \backslash W} \widetilde{I}_{\lambda} .
\end{gathered}
$$

Then it follows from [4] that $c_{\lambda}>0$ is a critical value of $\widetilde{I}_{\lambda}$ having a sign-changing critical point $u_{\lambda}$ at this critical value. From the construction of $c_{\lambda}$ and Lemma 1.4, we have $c_{\lambda} \leq b_{2, \lambda} \leq C_{*} \lambda^{-\frac{2}{\beta-2}}$. From Lemma 2.2 and for $\lambda$ large, the $u_{\lambda}$ 's are solutions of the original problem $\left(P_{\lambda}\right)$.

Proof of Theorem 2. To prove Theorem 2 we shall use the arguments in [3] and [4] to get solutions for $\left(\widetilde{P}_{\lambda}\right)$ first. In order to get estimates on the critical values we shall use the proofs in 3 for the existence and the proofs in [4 for the nodal property of the solutions. Fix an integer $k$. We shall show $\left(P_{\lambda}\right)$ has $k$ pairs of solutions for large $\lambda$, including $k-1$ pairs of nodal solutions.

Choose $R>0$ such that $\widetilde{I}_{\lambda}(u) \leq 0$ for all $u \in Y_{k}$ with $\|u\| \geq R$, and for all $\lambda \geq 1$. Let $D=B_{R} \cap Y_{k}$. Define $G=\{h \in C(D, E) \mid h$ is odd and $h(u)=u$, for $\|u\|=$ $R\}$. We denote the genus of a symmetric subset $A$ by $i(A)$ and, for $j=1, \ldots, k$, we let

$$
\begin{gathered}
\Gamma_{j}=\{h(\overline{D \backslash B}) \mid h \in G, k \geq j, i(B) \leq k-j\}, \\
c_{j, \lambda}=\inf _{A \in \Gamma_{j}} \sup _{u \in A} \widetilde{I}_{\lambda}(u) .
\end{gathered}
$$

Then, by [3] (Proposition 9.30, p. 58), and under our conditions on $f$, we have that $0<c_{1, \lambda} \leq c_{2, \lambda} \leq \cdots \leq c_{k, \lambda}$ are all critical values of $\widetilde{I}_{\lambda}$ and there are at least $k$ pairs of critical points at these critical values. Since $I d \in G$ we have

$$
c_{k, \lambda} \leq b_{k, \lambda} \leq C_{k} \lambda^{-\frac{2}{\beta-2}},
$$

where $b_{k, \lambda}$ is as in Lemma 1.4. Then, by Lemma 2.2 and for $\lambda$ large, these $k$ pairs of critical points are also solutions of the original problem.

Finally, we make use of the fact that $\inf _{s \in \mathbb{R}} \widetilde{F}^{\prime}(s)>-\infty$ (by construction) and follow the idea in 44 by restricting the above minimax procedure to $X$ and taking the maximum outside $W$. Choose $R>0$ such that $\widetilde{I}_{\lambda}(u) \leq 0$ for all $u \in Y_{k}$ with $\|u\| \geq R$, and for all $\lambda \geq 1$. Let $D=B_{R} \cap Y_{k}$ and define $G=\{h \in$ $C(D, X) \mid h$ is odd and $h(u)=u$, for $\|u\|=R\}$. We also denote the genus of a symmetric subset $A$ in $X$ by again $i(A)$ and, for $j=2, \ldots, k$, let

$$
\begin{gathered}
\Gamma_{j}=\{h(\overline{D \backslash B}) \mid h \in G, k \geq j, i(B) \leq k-j\}, \\
c_{j, \lambda}=\inf _{A \in \Gamma_{j}} \sup _{A \backslash W} \widetilde{I}_{\lambda}(u) .
\end{gathered}
$$

Then, by [4] (Theorem 2.3, p. 3214)) and, again, by the properties of $\widetilde{F}^{\prime}$, we have that $0<c_{2, \lambda} \leq c_{3, \lambda} \leq \cdots \leq c_{k, \lambda}$ are all critical values of $\widetilde{I}_{\lambda}$ and there are at least $(k-1)$ pairs of sign-changing critical points at these critical values. Also, since $I d \in G$, we have

$$
c_{k, \lambda} \leq b_{k, \lambda} \leq C_{k} \lambda^{-\frac{2}{\beta-2}}
$$


with $b_{k, \lambda}$ as in Lemma 1.4. Therefore, it follows from Lemma 2.2 and for $\lambda$ large that these $(k-1)$ pairs of sign-changing critical points are also solutions of the original problem.

Remark. We point out that all the above results are still true when $N=1,2$.

\section{REFERENCES}

[1] A. Ambrosetti, P. Rabinowitz, Dual Variational Methods in Critical Point Theory and Applications, J. Funct. Anal. 14 (1973), 349-381. MR 0370183 (51:6412)

[2] D.G. Costa, H. Tehrani, On the Sign of the Mountain Pass Solution, Nonlinear Analysis 44 (2001), 65-80. MR 1815692 (2002c:35111)

[3] P. Rabinowitz, Minimax Methods in Critical Point Theory with Applications to Differential Equations, CBMS Conf. Ser. in Math. 65, Amer. Math. Soc., 1986. MR 0845785 (87j:58024)

[4] S. Li, Z.-Q. Wang, Ljusternik-Schnirelman theory in partially ordered Hilbert spaces, Transactions of the American Mathematical Society, 354 (2002), 3207-3227. MR 1897397 (2003c:58009)

[5] Z.-Q. Wang, On a superlinear elliptic equation, Analyse Nonlinéaire 8 (1991), 43-58. MR 1094651 (92a:35064)

[6] M. Willem, Minimax Theorems, Birkhäuser, 1996. MR 1400007 (97h:58037)

Department of Mathematical Sciences, University of Nevada, Las Vegas, Nevada 89154

E-mail address: costa@unlv.nevada.edu

School of Mathematics and Computer Sciences, Fujian Normal University, Fuzhou 350007, People's Republic of China - and - Department of Mathematics and Statistics, Utah State University, Logan, Utah 84322

E-mail address: wang@math.usu.edu 